\title{
Correction to: Rich dynamics of non-toxic phytoplankton, toxic phytoplankton and zooplankton system with multiple gestation delays
}

\author{
Ashok Mondal $^{1}$ - A. K. Pal ${ }^{2}$. G. P. Samanta ${ }^{1}$
}

Published online: 5 February 2019

(c) Springer-Verlag GmbH Germany, part of Springer Nature 2019

\section{Correction to:}

International Journal of Dynamics and Control https://doi.org/10.1007/s40435-018-0501-4

In the original publication, Theorem 4.6 has been published incorrectly. The corrected theorem is given below:

Theorem 4.6 Suppose that system (3.2) satisfies the following conditions:

(i) $k_{2}-\frac{\beta_{21} k_{1}}{\alpha_{1}}>0$ and $/$ or $\frac{\gamma_{1} k_{1}}{\alpha_{1}}-\delta>0$;

(ii) $k_{1}-\frac{\beta_{12} k_{2}}{\alpha_{2}}>0$;

(iii) $\gamma_{1} \hat{X}_{1}-\delta-\gamma_{2} \hat{X}_{2}>0$;

(iv) $k_{2}-\beta_{21} \tilde{X}_{1}-\gamma_{2} \tilde{Y}>0$,

then system (3.2) is permanence.

Proof Let us consider the average Lyapunov function in the form $V\left(X_{1}, X_{2}, Y\right)=X_{1}^{\theta_{1}} X_{2}^{\theta_{2}} Y^{\theta_{3}}$ where each $\theta_{i}(i=$ $1,2,3)$ is assumed to be positive. In the interior of $\mathbb{R}_{+}^{3}$, we have

The original article can be found online at https://doi.org/10.1007/ s40435-018-0501-4.

Ashok Mondal

ashoke.2012@yahoo.com

A. K. Pal

akpal_2002@yahoo.co.in

G. P. Samanta

g_p_samanta@yahoo.co.uk; gpsamanta@math.iiests.ac.in

1 Department of Mathematics, Indian Institute of Engineering Science and Technology, Shibpur, Howrah 711 103, India

2 Department of Mathematics, S. A. Jaipuria College, Kolkata 700005, India

$$
\begin{aligned}
\frac{\dot{V}}{V}= & \psi\left(X_{1}, X_{2}, Y\right)=\theta_{1}\left[k_{1}-\alpha_{1} X_{1}-\beta_{12} X_{2}-\gamma_{1} Y\right] \\
& +\theta_{2}\left[k_{2}-\alpha_{2} X_{2}-\beta_{21} X_{1}-\gamma_{2} Y\right] \\
& +\theta_{3}\left[\gamma_{1} X_{1}-\delta-\gamma_{2} X_{2}\right] .
\end{aligned}
$$

To prove the permanence of the system, we shall have to show that $\psi\left(X_{1}, X_{2}, Y\right)>0$, for all boundary equilibria of the system. The values of $\psi\left(X_{1}, X_{2}, Y\right)$, at the boundary equilibria $E_{0}, E_{1}, E_{2}, E_{3}$ and $E_{4}$, are the following:

$$
\begin{aligned}
& E_{0}: \theta_{1} k_{1}+\theta_{2} k_{2}-\theta_{3} \delta . \\
& E_{1}: \theta_{2}\left(k_{2}-\frac{\beta_{21} k_{1}}{\alpha_{1}}\right)+\theta_{3}\left(\frac{\gamma_{1} k_{1}}{\alpha_{1}}-\delta\right) . \\
& E_{2}: \theta_{1}\left(k_{1}-\frac{\beta_{12} k_{2}}{\alpha_{2}}\right)+\theta_{3}\left(-\delta-\frac{\gamma_{2} k_{2}}{\alpha_{2}}\right) . \\
& E_{3}: \theta_{3}\left\{\gamma_{1} \hat{X}_{1}-\delta-\gamma_{2} \hat{X}_{2}\right\} . \\
& E_{4}: \theta_{2}\left\{k_{2}-\beta_{21} \tilde{X}_{1}-\gamma_{2} \tilde{Y}\right\} .
\end{aligned}
$$

Now, $\psi(0,0,0)>0$ is automatically satisfied for some $\theta_{i}>$ $0(i=1,2,3)$. Also, if the inequalities $(i)-(i v)$ hold, $\psi$ is positive at $E_{1}, E_{2}, E_{3}$ and $E_{4}$ for some $\theta_{i}>0(i=1,2,3)$. Therefore, system (3.2) is permanence [1] if the conditions (i) - (iv) are fulfilled. Hence the theorem.

\section{Reference}

1. Freedman HI, Ruan S (1995) Uniform persistence in functional differential equations. J Differ Equ 115:173-192 